\title{
Blast Domination Number for Transformation Graphs of Stars
}

\begin{abstract}
This article, muse on the widespread transformation graphs $G^{a b}$ and penetrate the related lexis of the Blast domination number of graphs, for all sorts of transformation graphs, $G^{a b}$ and their complement graphs, $\overline{G^{a b}}$ for Stars.

Keywords: Blast Domination Number of a graph, Triple connected domination Number of a graph, Triple connected graph.
\end{abstract}

\section{INTRODUCTION}

Graphs considered at this juncture are simple, connected, undirected graphs. For customary graph theoretic notation, we refer to West [1] whilst for jargon allied to domination in graphs we refer to Haynes et al. [10]. The concept of triple connected graph was introduced by J.Paulraj Joseph in [8]. Triple connected domination number of a graph was introduced by G.Mahadevan et.al in [2]. Recently, massive number of various triple connected domination parameters was introduced.

Provoked by all the above said triple connected domination parameters, in [3], freshly the conception of Blast domination number of a graph was introduced by A.Ahila et al., with relevance. The parameter is being studied over various concepts as in $[4,5,6,7]$. In this paper, and fragment, we flog out on the subject of the performance of the Blast domination number over the transformation graphs $[9,10$, 11] and their complement graphs of some standard graphs such as Stars. The brainwave of this exertion is a piece of the applications of such transformation graphs.

\section{PRELIMINARY DEFINITIONS}

A subset $\mathrm{S}$ of $\mathrm{V}$ of a non-trivial connected graph $\mathrm{G}$ is called a Blast dominating set (or) BD-set, if $\mathrm{S}$ is a connected dominating set and the induced sub graph $\langle V-S\rangle$ is triple connected. The least cardinality of all such Blast Dominating sets is called the Blast Domination Number (BDN) of $\mathrm{G}$ and is denoted by $\gamma_{c}^{t c}(G)$. Also, any Blast Dominating set with $\gamma_{c}^{t c}(G)$ vertices is called a $\gamma_{c}^{t c}$ - set of $\mathrm{G}$.

Let $\mathrm{G}(\mathrm{V}, \mathrm{E})$ be any simple, non-trivial connected graph. Let us denote a graph of order $p$ and size $q$, in short as a $(p, q)$ graph. Let $\alpha, \beta$ be two elements of $V(G) \cup E(G)$.We say the associability of $\alpha$ and $\beta$ is '十' if they are either adjacent or incident in $\mathrm{G}$, to boot is ' - '. Let $a b$ be a 2- permutation of the set, $\{t,-\}$. Now it is defined as $\alpha$ and $\beta$ matches up to the first structure of $a b$ if both $\alpha$

Revised Manuscript Received on December 15, 2019

* Correspondence Author

A. Ahila*, Mathematics, Kalasalingam Academy of Research and Education, Virudhunagar, India. Email: dr.aaasundar@gmail.com and $\beta$ are in $V(G)$, whereas $\alpha$ and $\beta$ matches up to the second structure of $a b$ if both $\alpha$ and $\beta$ are in $E(G)$. The generalized transformation graph $G^{a b}$ is being defined on the vertex set of $V(G) \cup E(G)$, two vertices $\alpha$ and $\beta$ of $G^{a b}$ are adjacent if and only if their associability in $\mathrm{G}$ is consistent with respect to the consequent term $a b$. Apparently, we get four graphical transformation of graphs, with regard to the four dissimilar 2- permutations of $\{+,-\}$, viz., $G^{++}, G^{+-}, G^{-+}$and $G^{--}$.

In other words, four graphical transformation of the generalized transformation graph $G^{a b}$ are the graphs whose vertex set is $V(G) \cup E(G)$ and $\alpha, \beta \in V\left(G^{a b}\right), \alpha$ and $\beta$ are adjacent in $G^{a b}$, if and only if either [*] or [**] holds.

[*] $-\alpha, \beta \in V(G), \alpha$ and $\beta$ are adjacent in $G$, when $a=+$, not adjacent when $a=-$

and $\quad, \quad$ are incident in $\mathrm{G}$, when

, not incident when

Therefore, the four graphical transformation of the generalized transformation graph are

and . The four graphical transformations are therefore defined as follows:

$$
\text { , and are adjacent in , if }
$$

1.

$-$

G

2.

$-$

incident in $\mathrm{G}$

3.

G

and , and are adjacent in $\mathrm{G}$ are incident in

\section{, and are adjacent in $\mathrm{G}$}

and are not
, and are not adjacent in are

incident in $\mathrm{G}$

4.

G 


\section{Blast Domination Number for Transformation Graphs of Stars}

and

are not

incident in $\mathrm{G}$

\section{CORE RESULTS}

Blast Domination Number over Transformation Graphs:

In this chapter, we attain the Blast domination numbers for both the generalized transformation graphs, $G^{a b}$ and their complement graphs, $\overline{G^{a b}}$ for stars.

Theorem 3.1: Let $G(V, E)$ be the star graph, $K_{1, p}$. Then the Blast domination number for all transformation graphs and their compliment graphs are as follows:

a) $\gamma_{c}^{t c}\left(K_{1, p}{ }^{++}\right)$and $\gamma_{c}^{t c}\left(\overline{K_{1, p}{ }^{++}}\right)$does not exist.

b) $\gamma_{c}^{t c}\left(K_{1, p}{ }^{+-}\right)=3, \forall p \geq 3$

$$
\gamma_{c}^{t c}\left(\overline{K_{1, p}{ }^{+-}}\right)=2, \forall p \geq 3 \text {. }
$$

c) $\gamma_{c}^{t c}\left(K_{1, p}{ }^{-+}\right)=\gamma_{c}^{t c}\left(\overline{K_{1, p}{ }^{-+}}\right)=3, \forall p \geq 3$.

d) $\gamma_{c}^{\text {tc }}\left(K_{1, p}{ }^{--}\right)$does not exist. Whereas, $\gamma_{c}^{t c}\left(\overline{K_{1, p}}\right)=1, \forall p \geq 3$

Proof: Let $G(V, E)$ be the star graph, $K_{1_{2} p}$. With respect to, $K_{1, p}{ }^{a b}$ and its complement graph, $\overline{K_{1, p}{ }^{a b}}$, the vertex set is $\left\{v_{1}, v_{2}, v_{3}, \ldots v_{p}, e_{12}, e_{13}, e_{14, \ldots .} e_{1(p-1)}, e_{1 p}\right\}$

Claim (a): Considering $K_{1, p}{ }^{++}$and its complement,

Blast domination number does not exist for $K_{1, p}{ }^{++}$, as the root vertex acts as a major vertex an if it is chosen as our BD-set, $S$, then the induced sub graph $\langle V-S\rangle$ is disconnected.

Furthermore, Blast domination number does not exist for $\overline{K_{1, p}{ }^{++}}$, for the reason that the root vertex remains isolated. View the result in Fig. 1, shown below:

Claim (b): Considering $K_{1, p}{ }^{+-}$and its complement,
The root vertex $v_{1}$ dominates all the other $\mathrm{p}$ vertices. Every $e_{1 j}$ dominates $v_{k}{ }^{\prime} s$ such that $k \neq 1$ or $j$. And so it is enough to choose $v_{2}$ and $v_{3}$, successively to dominate the rest vertices. Thus, our BD-set is $\left\{v_{1}, v_{2}, v_{3}\right\}$ and for this reason $\gamma_{c}^{t c}\left(K_{1, p}{ }^{+-}\right)=3, \forall p \geq 3$.

Whence, taking into consideration of the complement, $\overline{K_{1, p}{ }^{+-}}, v_{2}$ dominates all $v_{i}$ 's, $3 \leq i \leq p+1 ; e_{12}$ dominates all $e_{1 i}$ 's, $3 \leq i \leq p+1$, and $v_{1}$. So that the set $\left\{v_{2}, e_{12}\right\}$ satisfies our BD-set conditions and which brings out the least set. Thus, $\gamma_{c}^{t c}\left(\overline{K_{1, p}{ }^{+-}}\right)=2, \forall p \geq 3$. View the result inn Fig. 2, shown below:

Claim (c): Considering $K_{1, p}^{-+}$and its complement,

In view of, $K_{1, p}{ }^{-+},\left\{v_{1}, e_{12}, v_{2}\right\}$ is the least BD-set, for $v_{1}$ neighbors all $e_{1 i}$ 's whence $v_{2}$ neighbors all $v_{j}$ 's, for all the values of $2 \leq i \leq(p+1) ; 3 \leq j \leq(p+1)$. But $v_{1}$ and $v_{2}$ are not connected. Therefore, to make them connected and to safe $e_{12}$ from isolation, it is necessary to include, $e_{12}$. Thus, $\gamma_{c}^{\text {tc }}\left(K_{1_{1} p}{ }^{-+}\right)=3, \forall p \geq 3$. Allowing for, $\overline{K_{1, p}{ }^{-+}}, v_{1}$ neighbors all $v_{i}$ 's whence $v_{2}$ neighbors all $e_{1 j}$ 's except $e_{12}$ but $v_{3}$ adjacent $e_{12}$, for all the values of $2 \leq i \leq(p+1) ; 3 \leq j \leq(p+1)$. Hence $\gamma_{c}^{t c}-$ set of $\left(K_{1, p}{ }^{-+}\right)=\left\{v_{1}, v_{2}, v_{3}\right\}$. Thus, $\gamma_{c}^{t c}\left({K{ }_{1, p}{ }^{-+}}^{t}\right)=3, \forall p \geq 3$. View the result inn Fig. 3, shown below:

Claim (d): Considering $K_{1, p}{ }^{--}$and its complement,

Making an allowance for, $K_{1, p}{ }^{--}$, blast domination number does not exist, as the root vertex remains isolated.

In view of the complement graph of $K_{1, p}{ }^{--}$, we find that $v_{1}$ is a full vertex Hence, it is necessary to have $\left\{v_{1}\right\}$ as our Blast dominating set. Thus, $\gamma_{c}^{t c}\left(\overline{K_{1, p}}\right)=1, \forall p \geq 3$.

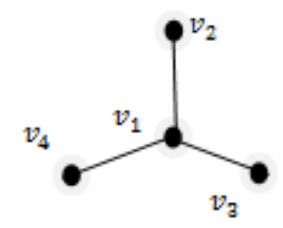

$K_{1,3}$

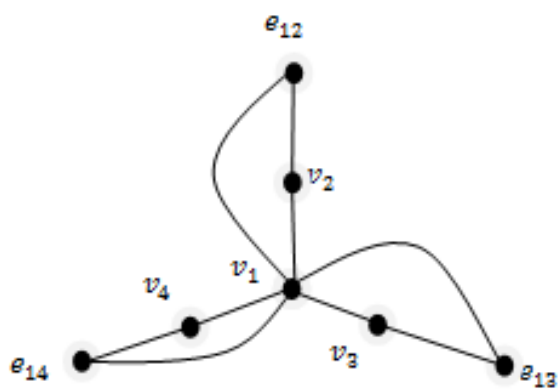

$K_{1,3}^{++}$

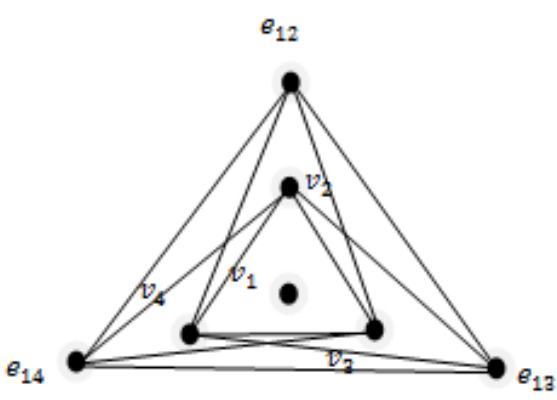

L.13 $_{1+3}^{+1}$

Fig. 1 


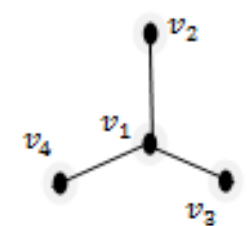

$K_{1,3}$

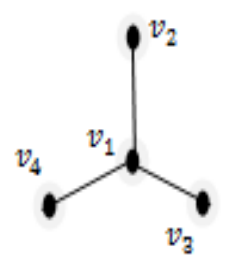

$K_{1,3}$

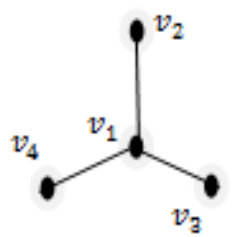

$K_{1,3}$

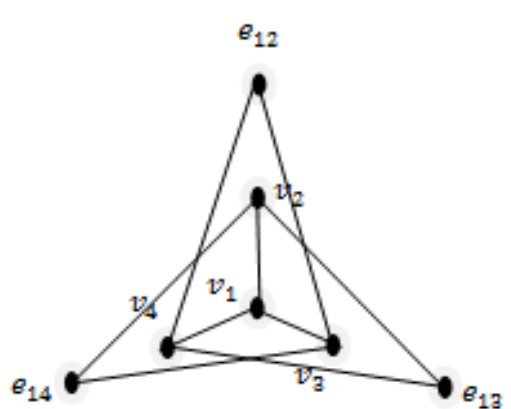

$K_{1, \overline{3}}^{+}$

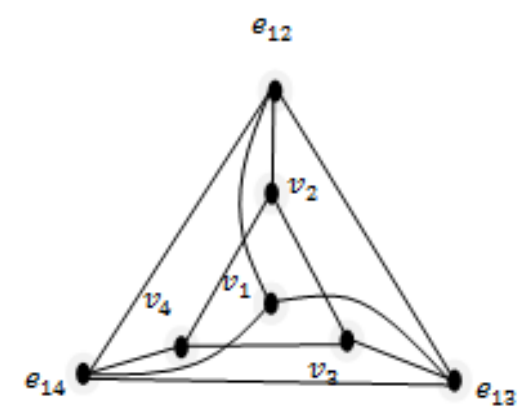

$\overline{K_{1,3}^{+}}$

Fig. 2

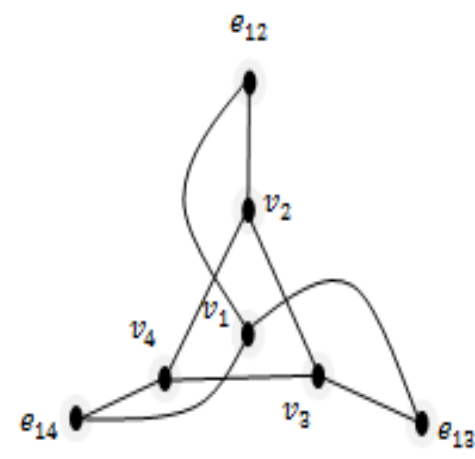

$K_{1,3}^{-+}$

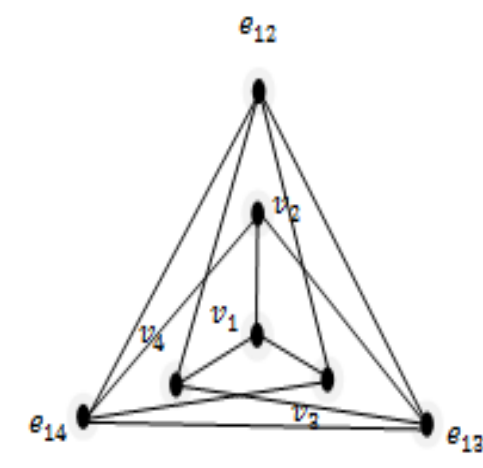

$\overline{\bar{K}_{13}^{-+}}$

Fig. 3

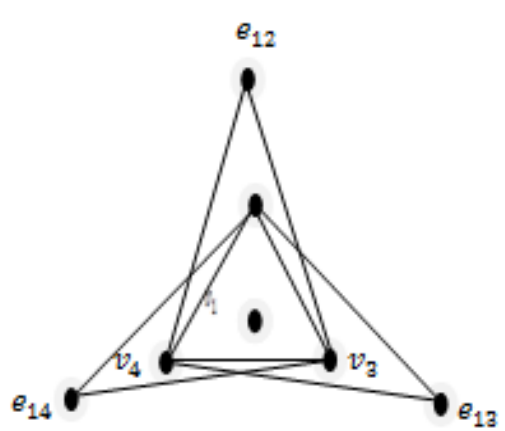

$K_{1, \overline{3}}^{-\overline{3}}$

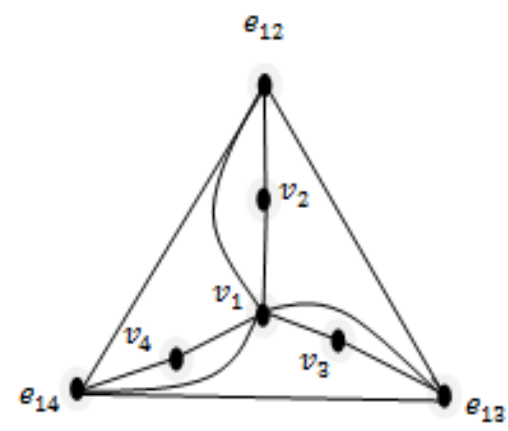

$\overline{K_{1,3}^{-}}$

Fig.4 


\section{CONCLUDING REMARKS}

The domination and inclination of network are the two noteworthy factors for the network structure. We have introduced an imperative measure of liability called Blast domination number. Here we have investigated the Blast domination number for transformation graphs of stars. The results reported here are sure to throw some glow in the track to work the same in larger graphs obtained from the given graph.

\section{REFERENCES}

1. D.B. West, Introduction To Graph Theory, 2/e, Prentice-Hall, New Delhi, India, 2003.

2. G.Mahadevan, A.Selvam, J.Paulraj Joseph and T.Subramanian, Triple connected domination number of a graph, International Journal of Mathematical Combinatorics, Vol.3 (2012), 93 - 104.

3. G.Mahadevan, A. Ahila and S. Avadayappan, Blast domination number of a graph, Middle East J. Sci. Res.,25(5) (2017), 977-981.

4. G. G.Mahadevan, A..Ahila and S.Avaayappan, Blast Domination Number for $\vartheta$ - Obrazom, International Journal of Pure an Applied Mathematics, Volume 118, No.7, 2018, 111 - 117.

5. G.Mahadevan, A.Ahila, Selvam Avadayappan, Blast Domination Number of a graph Further Results, International Journal of Computational and Applied Mathematics, (2017) Volume 12, Number $1,307-312$.

6. G.Mahadevan, A.Ahila, Selvam Avadayappan, Blast Domination Number For Central and Total Graph of Star, Global Journal of Pure and Applied Mathematics, (2017) Volume 13, Number 2, 984 - 990.

7. G.Mahadevan, A.Ahila, Selvam Avadayappan, Blast Domination Number For Square and Cube Graphs of Graphs, in the Contemporary Studies in Discrete Mathematics - CSDM journal, Vol. 1, No. 1, 2017, pp. 31-35.

8. J.Paulraj Joseph, M.K.Angel Jebitha, P.Chithra Devi and G.Sudhana, Triple connected graphs, Indian Journal of Mathematics and Mathematical Sciences, Vol.8, No.I(2012),61-75.

9. Lei $\mathrm{Yi}$, Baoyindureng $\mathrm{Wu}$, The Transformation Graph $\mathrm{G}^{+\mathbf{f}^{-}}$, Australasian Journal of Combinatorics, Volume 44 (2009), Pages 37 42.

10. T. Haynes, S. Hedetniemi, and P. Slater, Fundamentals of Domination in Graphs, Marcel Dekker, New York, NY, USA, 1998.

11. V.R.Kulli, The Entire Edge Dominating Graph, in Advances in Domination Theory I, V.R.Kulli, ed., Vishwa International Pubications, Gulbarga, India, 155 (2012).

\section{AUTHORS PROFILE}

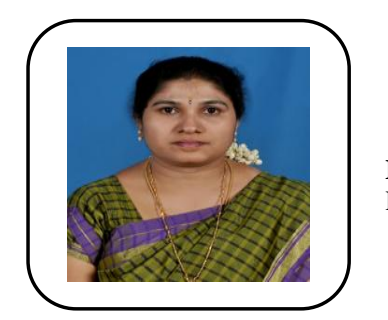

Ms. A. AHILA.,

M.Sc., M.Phil., B.Ed., [Ph.D]., Professor of Mathematics.

> Publications - 9 published, 3on process.

$>$ Research - Introduced and working on the new parameter Blast Dominatin Number of a Graph,

$>$ Memberships - Life time member in ISTE and IAENG, BOM KARE

$>$ Achievements - 21 years of teaching experience, Reviewer CSDM, Best Paper Award winner, Proud Rotarian. 